\title{
Using precise boron neutron capture therapy as a salvage treatment for pediatric patients with recurrent brain tumors
}

\author{
Yi-Wei Chen ${ }^{1,2}$, Tien-Li Lan ${ }^{1}$, Yi-Yen Lee ${ }^{3}$, Ko-Han Lin ${ }^{4}$, Muh-Lii Liang ${ }^{3}$, Hsin-Hung Chen ${ }^{3}$, \\ Po-Shen Pan ${ }^{5}$, Feng-Chi Chang ${ }^{6}$, Chih-Chun $\mathrm{Wu}^{6}$, Shih-Chieh Lin ${ }^{7}$, Yu-Mei Kang ${ }^{1}$, Jia-Cheng Lee ${ }^{1,2}$, \\ Hong-Ming Liu ${ }^{8}$, Kuan-Hsuan Chen ${ }^{9}$, Jen-Kun Chen ${ }^{10}$, Chun-Fu Lin ${ }^{3}$, Yu-Cheng Kuo ${ }^{11}$, \\ Shih-Ming Hsu ${ }^{12}$, Wen-Sheng Huang ${ }^{4}$, Fong-In Chou ${ }^{8}$
}

${ }^{1}$ Division of Radiation Oncology, Department of Oncology, Taipei Veterans General Hospital, Taipei, Taiwan; ${ }^{2}$ Department of Medical Imaging and Radiological Technology, Yuanpei University of Medical Technology, Hsinchu City, Taiwan; ${ }^{3}$ Department of Pediatric Neurosurgery, Neurological Institute, Taipei Veterans General Hospital, Taipei, Taiwan; ${ }^{4}$ Department of Nuclear Medicine, Taipei Veterans General Hospital, Taipei, Taiwan; ${ }^{5}$ Department of Chemistry, Tamkang University, Taipei, Taiwan; ${ }^{6}$ Department of Radiology, Taipei Veterans General Hospital, Taipei, Taiwan; ${ }^{7}$ Department of Pathology and Laboratory Medicine, Taipei Veterans General Hospital, Taipei, Taiwan; ${ }^{8}$ Nuclear Science and Technology Development Center, National Tsing-Hua University; Hsinchu City, Taiwan; ${ }^{9}$ Department of Pharmacy, Taipei Veterans General Hospital, Taipei, Taiwan; ${ }^{10}$ Institute of Biomedical Engineering and Nanomedicine, National Health Research Institutes, Miaoli, Taiwan; ${ }^{11}$ Department of Radiation Oncology, China Medical University Hospital, Taichung, Taiwan; ${ }^{12}$ Department of Biomedical Imaging and Radiological Sciences, National YangMing University, Taipei, Taiwan

Contributions: (I) Conception and design: YW Chen; (II) Administrative support: TL Lan; (III) Provision of study material or patients: YW Chen, ML Liang, HH Chen; (IV) Collection and assembly of data: YW Chen; (V) Data analysis and interpretation: YW Chen; (VI) Manuscript writing: All authors; (VII) Final approval of manuscript: All authors.

Correspondence to: Yi-Wei Chen, MD, PhD. Department of Oncology, Taipei Veterans General Hospital, No. 201, Sec. 2, Shi-Pai Rd., Taipei City, Taiwan. Email: chenyw@vghtpe.gov.tw.

Background: Boron neutron capture therapy (BNCT) is a precise targeted particle therapy. Its principal purpose is to specifically administer boron-10-containing drugs to tumor cells and subsequently irradiate them with thermal neutrons. This highly explosive tumor destruction method exclusively attacks tumor cells that uptake boron-10-containing drugs, while minimizing damage to the surrounding normal cells without drug uptake, thereby making it an ideal option for the precise treatment of pediatric patients with recurrent brain tumors.

Methods: Since March 2017, Taipei Veterans General Hospital collaborated with the Nuclear Science and Technology Development Center of National Tsing-Hua University to develop a salvage BNCT for recurrent tumors in the central nervous system. BNCT was delivered to patients using the research reactor of Tsing-Hua University. As of January 2020, a total of nine BNCT treatments (10 sites) have been performed on five pediatric patients with recurrent brain tumors.

Results: The diagnoses of all five patients were recurrent glioblastomas, two of which developed from the brainstem. The median follow-up duration was 5.3 months. After BNCT, one site had complete response, and three had partial response. The median progression-free interval was 3.8 months. None of the five patients showed severe normal brain tissue necrosis nor serious complications after treatment.

Conclusions: Pediatric patients are a vulnerable group with medical needs that are distinct from those of adult patients. BNCT is a precise cancer treatment for pediatric recurrent brain tumors with less normal toxicities and high tumor response.

Keywords: Boron neutron capture therapy (BNCT); glioblastoma; pediatric; precision; radiotherapy

Received: 25 February 2020; Accepted: 16 October 2020; Published: 30 December 2020.

doi: 10.21037/tro-20-20

View this article at: http://dx.doi.org/10.21037/tro-20-20 


\section{Introduction}

According to the data from the Childhood Cancer Foundation, Taiwan, brain tumor has the highest incidence among solid tumors in children, with approximately 100 150 cases annually in Taiwan (1). This number has gradually declined recently due to fewer births. Unlike those in adult patients, the primary brain tumor types in pediatric patients in Taiwan include medulloblastoma, germ cell tumors, and malignant gliomas (2). In addition, because of the variability in brain tumor types, the treatment strategies and prognosis of pediatric patients significantly differ from those of adult patients. Traditional standard management mainly includes radical surgery, followed by postoperative radiotherapy and chemotherapy. Although most pediatric patients with brain tumor demonstrate better prognosis than adult patients, they are more vulnerable to complications. The current standard treatment for these patients is often accompanied by related adverse effects. Particularly, after radiotherapy, it is possible that acute intracranial pressure changes that result in symptoms, such as nausea, vomiting, and headache, are often observed. Others include skin reactions in the irradiated area that lead to alopecia and chronic longterm effects, including deterioration of neurocognitive functions and growth restrictions due to imbalanced growth hormone levels. Therefore, alleviation of various treatment complications is critical for pediatric patients with brain tumors. Despite better prognosis compared to adult patients after comprehensive treatment management, pediatric patients can still have recurrences of highly malignant brain tumors, such as glioblastoma. Once this type of tumor recurs, the prognosis is usually unfavorable. Other than repeating craniotomy for tumor resection, the remaining treatment methods can only delay tumor progression rather than treat it.

Boron neutron capture therapy (BNCT) is an advantageous treatment option with strong biological effects. Since its first clinical trial in human patients with malignant brain tumors by a research team in the United States in the 1950s (3), BNCT has become an area of intense research focus worldwide. As a special medical technology, the success of BNCT relies on the integrated multidisciplinary development of clinical treatment strategies against the tumor, boron-containing drug chemistry, radiobiology, medical physics, and neutron nuclear engineering technology (4). The principle underlying this targeted particle therapy involves the specific administration of boron-10-containing drugs to the tumor, thereby labeling its location. Subsequently, by irradiating the tumor with thermal neutrons at low doses, boron-10, which has a strong capture and binding ability, efficiently binds to thermal neutrons and is cleaved into an $\alpha$-particle and recoiling lithium nucleus. These two particles not only are capable of effectively destroying tumor cells but also have a limited reaction range of $5-10 \mu \mathrm{m}$. Therefore, treatment is restricted within the tumor cells, whereby the surrounding normal tissue that does not absorb boron-10 remains unaffected, making it an ideal treatment option against tumors (Figure 1). Although, presently, BNCT is mainly used against brain tumors in adult patients (5-7), based on its treatment principle and mechanism, we believe that there are numerous advantages and great potential for the treatment of pediatric patients with brain tumor. In this article, we will share the experience of treating five pediatric patients (a total of ten sites irradiated with BNCT) with recurrent brain tumors. We will focus on not only the treatment responses, but also the patient's condition after the treatment as well as the normal tissue adverse effects. We present the following article in accordance with the STROBE reporting checklist (available at http://dx.doi. org/10.21037/tro-20-20).

\section{Methods}

In March 2017, Taipei Veterans General Hospital initiated a collaboration with the Nuclear Science and Technology Development Center of National Tsing-Hua University to use the Tsing-Hua Open Pool Reactor (THOR) to provide salvage BNCT as a compassionate treatment method. The study was approved by the institutional review board (IRB) of Taipei Veterans General Hospital and Taiwan Food and Drug Administration, Ministry of Health and Welfare R.O.C. Of 40 patients who completed BNCT, five were pediatric patients with recurrent brain tumors. The primary tumors of these five patients were all previously treated with surgery, radiotherapy, and chemotherapy. Upon tumor recurrence, evaluation by the specialized pediatric neuro-oncology team of our hospital suggested that no other active treatment modality was suitable. Therefore, remedial medical care was provided to the five pediatric patients through special medical applications. The tumor pathologies of all five patients were highly malignant glioblastoma, mainly WHO grade IV (8). The glioblastomas of the first two cases were possibly associated with previous radiotherapy and suspected to be radiation-induced malignancy. Two other patients, who were previously 
treated with standard radiotherapy, were diagnosed with diffuse midline glioma (8). Treatment options available for these two patients upon recurrence were limited.

All pediatric patients underwent 4-borono-2 ${ }^{18} \mathrm{~F}$-fluorophenylalanine-positron emission tomography (FBPAPET) 1-2 weeks before salvage BNCT was arranged (Figure $2 A$ ). The purpose of the examination was to label

\section{Mechanism of Boron Neutron Capture Therapy}
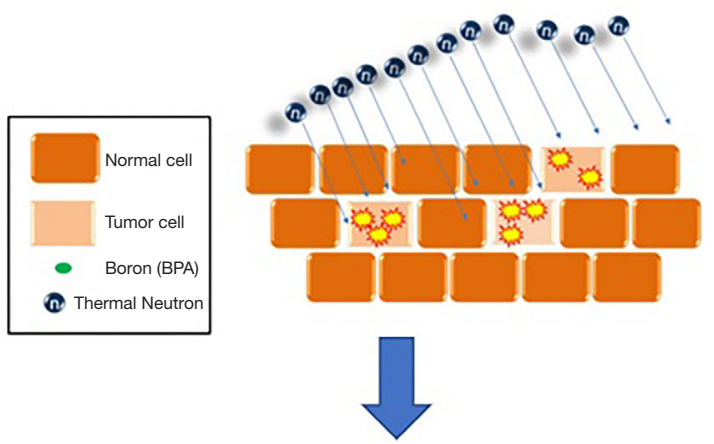

Tumor cells are selectively destroyed without damage of adjacent normal tissue.

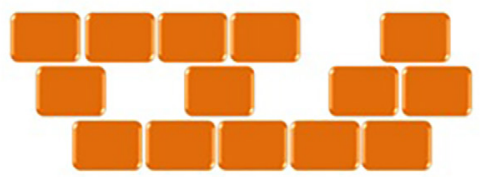

Figure 1 Schematic diagram of BNCT's mechanism. BNCT, boron neutron capture therapy. boron-10-containing drug 4-borono-2 $-{ }^{18} \mathrm{~F}$-fluoro-Lphenylalanine (BPA) with radioisotope fluorine-18 to display the distribution of the boron-containing drug via a PET scanner. According to the international BNCT clinical research guidelines, if the ratio between the standardized uptake value of the tumor and that of the surrounding normal tissue [tumor uptake/normal tissue uptake (T/ $\mathrm{N}$ ratio)] or blood pool [tumor uptake/blood pool uptake (T/B ratio)] is $>2.5$, the patient is considered suitable for BNCT $(4,9)$. In our study, the median $\mathrm{T} / \mathrm{N}$ and $\mathrm{T} / \mathrm{B}$ ratios of the five patients before treatment were 2.32 and 2.50 , respectively, which met the requirements of BNCT (4).

Treatments were arranged for a total of 10 tumor areas in the five patients (Table 1). For the second patient, two sites were simultaneously irradiated during one fraction. Three patients received multiple BNCTs (the first patient received two BNCTs at different sites; the fourth patient received three BNCTs; the fifth patient with brain stem tumors, who came from mainland China, received two planned BNCTs). Patients were admitted to the pediatric neuro-oncology ward at Taipei Veterans General Hospital the day before treatment. On the day of the procedure, the patients were transferred by an ambulance from Taipei Veterans General Hospital to THOR located at the Nuclear Science and Technology Development Center, National Tsing-Hua University. To satisfy the medical requirements of BNCT, THOR was upgraded since 1990s to deliver "epithermal neutrons", thereby achieving the effect of neutron irradiation on deeper brain tumors (approximately 7-8 cm) than the depth previous "thermal neutron" reaches (10).
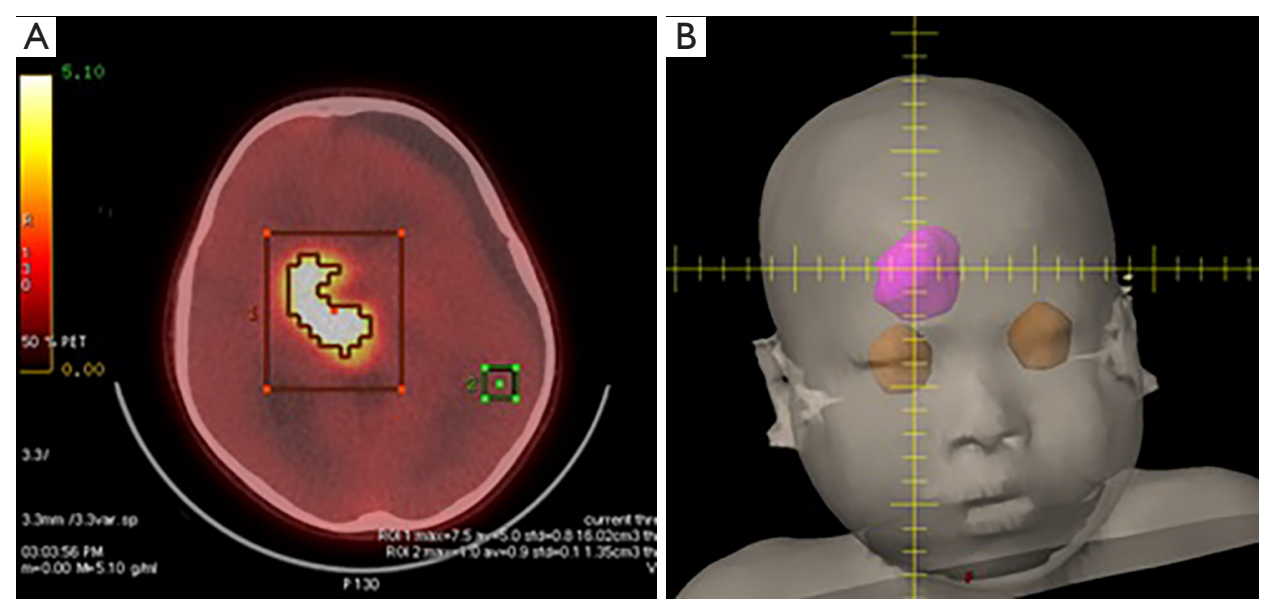

Figure 2 Pre-BNCT evaluation for patient No. 1. (A) High BPA uptake of patient No. 1 detected by FBPA-PET. (B) Simulation for BNCT of patient No. 1. BNCT, boron neutron capture therapy; FBPA-PET, 4-borono-2- ${ }^{18}$ F-fluoro-phenylalanine-positron emission tomography. 


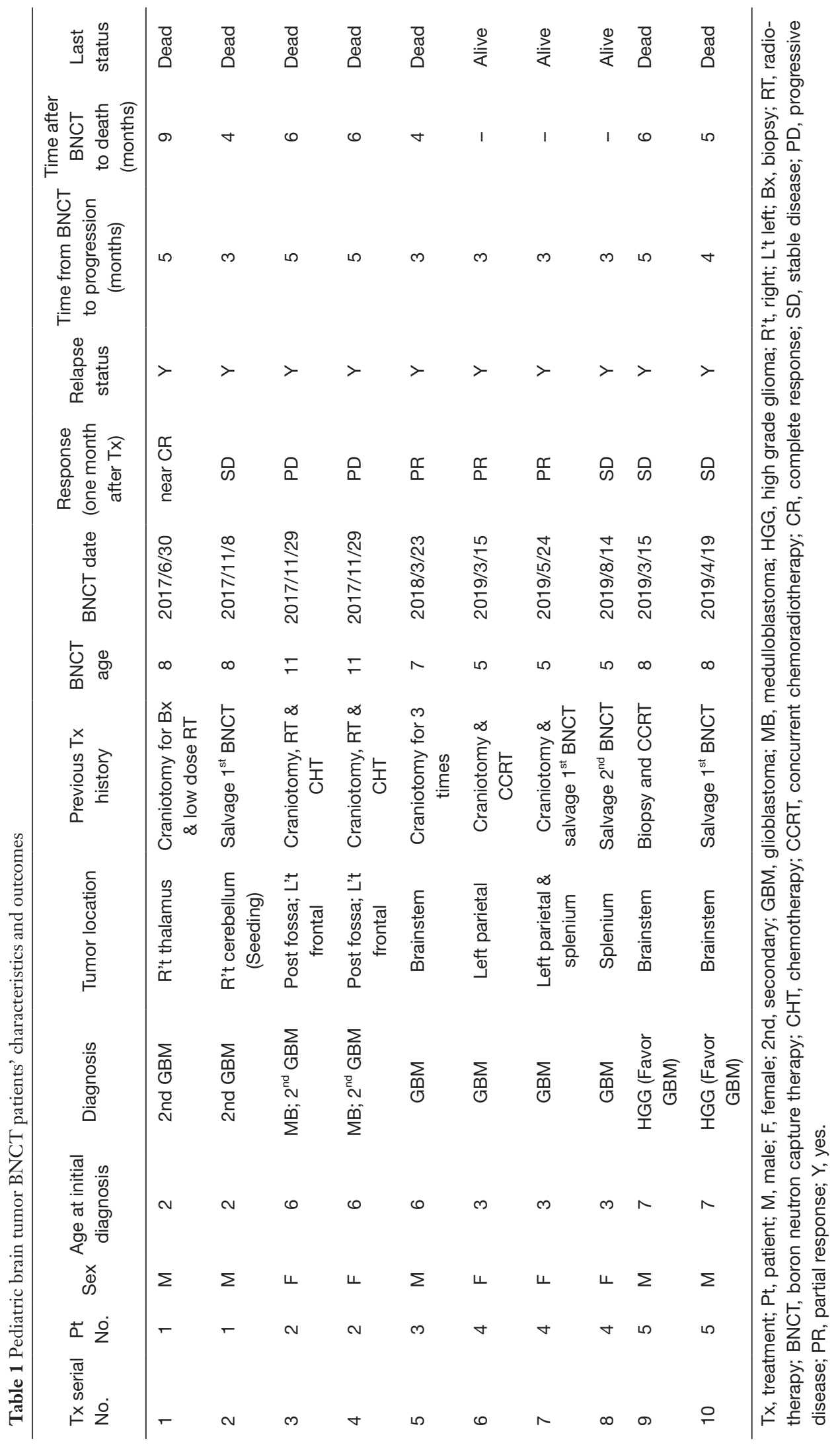


Before BNCT, a treatment plan was generated using the treatment planning system of Tsing-Hua University, during which the dose prescription for the tumor and dose limits for normal tissue were specified (Figure 2B). Then, the plan approved by the treatment team was delivered on the day of the procedure. Before neutron irradiation, the patient received continuous infusion of a boron-10-containing drug, BPA. This drug, synthetized and prepared by Taiwan Biotech Company, fulfilled Taiwan's Pharmaceutical Good Manufacturing Practice regulations. With a structure similar to that of phenylalanine, which is an amino acid essential to the human body, BPA was prepared by replacing the collateral structure of the phenyl ring with boric acid containing boron-10. Since tumor cells demand more essential amino acids than normal cells for proliferation, they absorb a large amount of BPA, which subsequently binds with thermal neutrons and produces destructive reactions. THOR is a uranium-235 circulating pool reactor with a standard power of 2.0 MW and TRIGA as the reaction fuel. Its optimal neutron flux, which satisfies the international requirements of BNCT $\left(>10^{9} \mathrm{n} \mathrm{cm}^{-2} \cdot \mathrm{s}^{-1}\right)$ (4), reduced the irradiation time of patients to approximately only 20 min each. During irradiation, patients will not have discomfort, such as burning sensation and pain. Before irradiation, patients receive continuous infusion of BPA at a dose of $450 \mathrm{mg} / \mathrm{kg}$ of body weight (i.e., for each kilogram of body weight, the patient must receive $450 \mathrm{mg}$ of BPA injection). The infusion formulation was designed for full infusion during the first $2 \mathrm{~h}$, followed by half infusion (infusion formulation was $180 \rightarrow 180 \rightarrow 90 \mathrm{mg} / \mathrm{kg}$ of body weight) during neutron irradiation. This formulation was proposed by the BNCT research team of Kyoto University (Institute for Integrated Radiation and Nuclear Science; KURNS), with the expectation of maintaining a constant concentration of boron-10-containing drug in the patient's blood, thereby ensuring that the treatment dose of the patient's tumor remains stable during irradiation with epithermal neutron (5).

To assist younger patients to complete the treatment, our team adopted the anesthesia-free sedation strategy generally used in pediatric patients at Taipei Veterans General Hospital. During the irradiation process, animated cartoons were projected onto the ceiling of the treatment bunker to reduce the possible anxiety of pediatric patients (11). During the study, the five pediatric patients successfully completed irradiation of all 10 areas without any anesthesia interventions.

All patients were followed until October 31, 2019 or the date of death. The magnetic resonance image-based RANO criteria were adopted to evaluate tumor responses on postoperative images (12). Alternatively, the adverse effects on normal tissue after treatment were assessed using CTCAE version 5.0 (13). The progression-free period was defined as date after BNCT to the date of tumor progression, death, or last follow-up. All collected data were reviewed and statistically analyzed using the statistical software $\mathrm{R}$ project ( $\mathrm{R}$ version 3.4 .3 ; http://rproject. org). The study was conducted in accordance with the Declaration of Helsinki (as revised in 2013). The study was approved by institutional review board of Taipei Veterans General Hospital with the registration number of 2017 05-E01A, 2017-11-E02B, 2018-03-E02C, 2019-01-E02B, 2019-03-E01B, and informed consent was taken from all the patients

\section{Results}

All five pediatric patients had recurrent malignant intracranial glioblastomas. The median age of patients was 8 years. The median follow-up duration after BNCT was 5.3 months (range, 3.1-9.7 months). After treatment, four patients died, and one patient survived.

The fourth patient received three treatments. She had recurrent left parietal glioblastoma. The tumor was initially detected when she was aged nearly 3 years. Despite previous radical surgery followed by standard postoperative radiotherapy and chemotherapy, the tumor continued to relapse. Although, upon the first recurrence, the lesion was removed by remedial surgery, at the beginning of 2019 , a large number of tumors rapidly relapsed, making it impossible to control the disease through radical surgery. After the application was approved, the first BNCT was performed on the patient in March 2019. Following treatment, significant tumor regression was observed (Figure 3), and concurrently, the patient demonstrated normal limb movements and superior quality of life. Unfortunately, after treatment, the tumor tissue still gradually crossed over to the contralateral brain lobe through the splenium of the corpus callosum. Although the following two adjuvant BNCTs could not prevent the continuous tumor progression, tumor control was achieved for $>6$ months.

An analysis was conducted on the BNCT outcome of the five pediatric patients with brain tumor. The median tumor volume that was irradiated was $34.3 \mathrm{~mL}$ (range, $8.1-$ $41.5 \mathrm{~mL}$ ). Before treatment, the boron concentration in the 


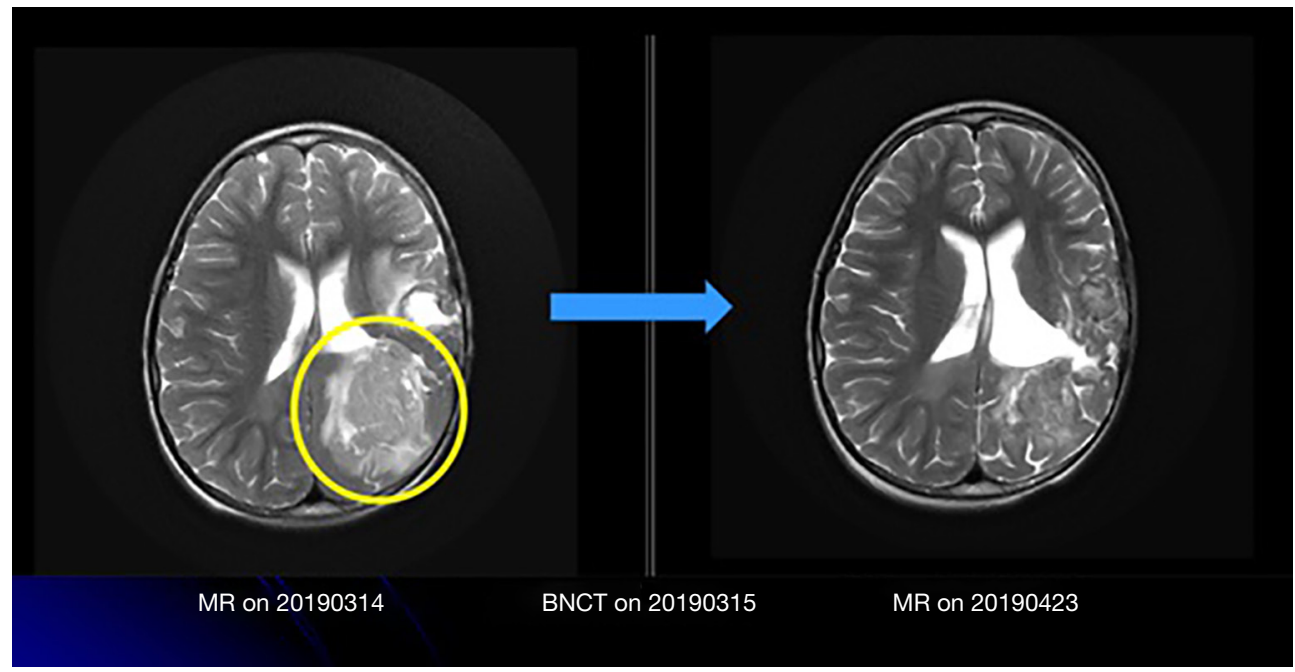

Figure 3 Significant tumor regression in patient No. 4 after BNCT. BNCT, boron neutron capture therapy.

blood was measured by inductively coupled plasma atomic emission spectroscopy, the median of which was $24.0 \mathrm{ppm}$ (range, 18.9-34.0 ppm).

The median prescription dose of the treatment was 15.7 Gy-E (range, 6.3-29.0 Gy-E). The median maximum dose was 25.0 Gy-E (range, 7.5-39.1 Gy-E), while the median minimum dose was 7.3 Gy-E (range, 5.0-21.8 Gy-E). In the follow-up images acquired between 1 and 3 months after treatment, of ten irradiated tumor sites, one showed a "near complete response" status, three showed a "partial response" status, four showed a "stable disease" status, and two showed a "progressive disease" status (Table 2).

No patient developed severe complications within one week of treatment other than occasional slight changes in intracranial pressure, causing head discomfort. These minor symptoms were effectively alleviated through steroid administration. Although all five pediatric patients showed obvious hair losses at the irradiated area, no severe skin damage was observed. Routine bevacizumab antiangiogenesis targeted therapy was delivered to all patients after treatment based on the recommendations by Miyatake et al. (14). None of the five patients showed severe normal brain tissue necrosis after treatment.

With the median progression-free period of 3.8 months, tumor responses were observed in all five patients. Four patients subsequently died due to disease progression.

\section{Discussion}

Glioblastoma, classified as grade IV by the WHO, is a disease with highly dismal prognosis (8) for both adult and pediatric patients. Even after traditional complete tumor resection followed by postoperative radiotherapy and chemotherapy, the overall survival period is $14-16$ months (15). In addition, upon tumor recurrence, no standard remedial treatment is effective, making the treatment of glioblastoma a major challenge in clinical medicine. Despite the emergence of multiple novel treatment modalities, such as those reported by Gliadel Wafer (16) and TTF (17), patient survival has barely improved. Pediatric patients are a particularly fragile group. In these patients, the design and management of all treatments should be carefully performed to prevent possible complications. On this basis, precision medicine is the key to enhancing the vulnerable situation of pediatric patients.

The idea of BNCT was proposed in the 1930s by radiation oncology researchers, who suggested that only tumor cells that absorbed boron-10-containing drugs would be damaged by the radiation particles with high linear energy transfer. As it prevents damage to the surrounding normal tissue, BNCT is an ideal biologically targeting particle therapy technique $(18,19)$. In addition to multiple clinical trials involving the use of BNCT on adult patients with malignant brain tumors $(20,21)$, Nakagawa et al. reported the treatment of 23 children and adolescents aged $<15$ years with various types of malignant brain tumors (22). The treatment was conducted on a Japan Atomic Energy Agency (JAEA) atomic reactor using sodium borocaptate (BSH) as the boron-10-containing drug. This empirical study reported that, in patients with grade IV glioblastoma, 


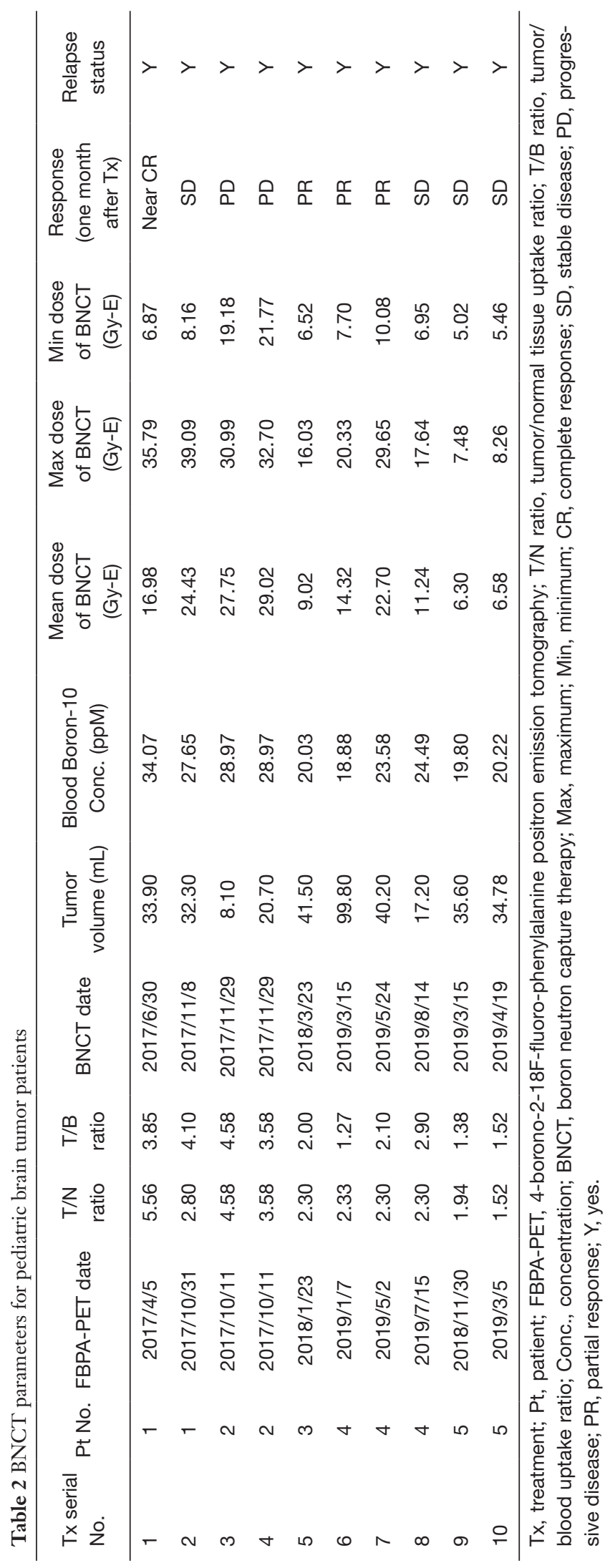

the tumor relapsed and metastasized to different areas of the spinal cord after BNCT, and the treatment achieved stable tumor control for a limited period. In contrast, for the seven remaining patients with WHO grade III malignant gliomas, complete tumor response was achieved, and no recurrence was reported. Our study reported the treatment of pediatric patients with recurrent glioblastoma using BPA-based BNCT for the first time worldwide. During the study, the amino acid structural drug, BPA, was adopted as the boron10 -containing drug. The structure and drug absorption dynamics of BPA are different from those of BSH (18). According to the international clinical requirements for BNCT, before neutron irradiation, the boron concentration in the blood should reach approximately $25 \mathrm{ppm}$ (4); an adequate boron concentration in the blood ensures sufficient boron-10-containing drug uptake by the tumor (the T/N and T/B ratios were calculated to derive the true boron concentration of the tumor with assistance of FBPAPET). During the BNCT dose calculation, the largest dose contribution was found to come from the response to the boron-10-containing drug (other sources included doses from the neutron itself and the $\gamma$-ray). The dose calculation of BNCT significantly differs from that of traditional photon therapy, as differences in the uptake of boroncontaining drugs between tumor cells and various normal tissues must be considered. Therefore, prior to assessing BNCT dose distributions, relevant biological calculations must be performed to clarify the drug uptake capacities of various types of cells $(18,23)$ so that the dose estimation is accurate.

Another important observation noted in this study was that, in addition to improvements in tumor response, none of the pediatric patients had any severe adverse effects. During traditional radiotherapy courses, one of the greatest risks of treatment is changes in intracranial pressure. On the contrary, after BNCT, no patient developed severe and acute changes in intracranial pressure that required emergency medical treatment. We have even encountered a patient who not only recovered from neurological deficits after BNCT but was able to return to daily life and even resume work after treatment. It is also worth mentioning that pediatric patients with this type of brain tumor all previously received full doses of photon radiotherapy. When BNCT is performed in addition to these treatments, the impact of accumulated doses should be carefully evaluated. Presently, as the principles behind the dose calculations of BNCT and photon therapy are different, doses from these two treatment modalities cannot be 
directly summed. However, as these two modalities are now often organized in a sequential order, the interval between the two treatment courses should be carefully assessed and arranged with a gap of at least half a year. In addition, the tumor dose prescription for BNCT adopted by our team was slightly lower than that in the internationally published dose scheme. This is mainly because our study was Taiwan's first treatment of pediatric patients with brain tumor using BNCT; therefore, we prioritized safety so as to achieve a balance between treatment efficacy and normal tissue toxicity. Moreover, during our study, pediatric patients with brain tumor were regularly administered a targeted drug, bevacizumab, after BNCT. As a novel antiangiogenic drug, bevacizumab was originally applied as a remedial treatment to suppress tumor changes (24). However, clinical BNCT trials discovered that bevacizumab could also inhibit normal brain tissue from radiotherapy-induced necrosis (including BNCT) and, hence, recommend its routine management after $\operatorname{BNCT}(14,25)$.

Lastly, FBPA-PET is an essential evaluator of whether BNCT can successfully assist the management of pediatric patients. By labeling the therapeutic BPA drug with the F-18 isotope, FBPA-PET can accurately determine the uptake of BPA by the tumor. This mode of using drugs to assist both treatment and diagnosis is referred to as theranostics (i.e., therapy + diagnostics) in nuclear medicine technology (26). During this process, our colleagues in the Department of Nuclear Medicine not only detected tumor uptake but also compared it with that of the normal tissue and blood (the cardiac ventricle was selected as the blood pool in the study) so that the ratios between these processes could be used to subsequently estimate the boron concentration of the tumor. This nuclear medicine technology is an indispensable factor for the success of BNCT (27).

Recurrent brain tumors in pediatric patients, especially glioblastoma, are severely debilitating. Previously, if remedial surgery could not be performed, the pediatric patient would lose their life within a short timeframe. In a previous population-based study by the Johns Hopkins group investigating the cancer-specific mortality of pediatric high grade glioma using Surveillance Epidemiology and End Results (SEER) database, they included 154 patients with high grade glioma (28). The results showed a median survival of 10 months, while radiation therapy was associated with a survival benefit at 6 and 9 months, but not at 1 or 2 years. BNCT is a novel, precise targeted therapy. By specifically targeting brain tumor cells with boron-10containing drug uptake and producing destructive effects with high precision, BNCT can fully satisfy the treatment demands of vulnerable pediatric patients. Although the development of a new treatment modality against tumors is time consuming, continuous breakthroughs in various fields involving the technology will conceivably result in BNCT becoming feasible for more pediatric patients with the same type of brain tumors, helping them achieve better treatment outcomes.

\section{Acknowledgments}

The research group would like to express utmost gratitude to the following researchers who provided assistance to the study: Prof. Yen-Wen Liu Hsieh (providing commercialized THORplan as a treatment planning system); Taiwan Biotech Company for manufacturing of L-BPA; Chi-Wei Chang, Nuclear Pharmacist of Department of Nuclear Medicine, Taipei Veterans General Hospital; and study nurse Pei-Yu Lai and research assistant Meng-Hsuan Lin, Research Center for Boron Neutron Capture Therapy at Tamkang University.

Funding: None.

\section{Footnote}

Provenance and Peer Review: This article was commissioned by the Guest Editors (Chen-Hsi Hsieh, Hsin-Hua Nien, and Yu-Ming Huang) for the series "Precision Medicine for Cancer" published in Therapeutic Radiology and Oncology. The article has undergone external peer review.

Reporting Checklist: The authors have completed the STROBE reporting checklist. Available at http://dx. doi. org/10. 21037/tro-20-20.

Conflicts of Interest: All authors have completed the ICMJE uniform disclosure form (available at http://dx.doi. org/10.21037/tro-20-20). The series "Precision Medicine for Cancer" was commissioned by the editorial office without any funding or sponsorship. YWC serves as an unpaid editorial board member of Therapeutic Radiology and Oncology from Apr 2020 to Mar 2022. The authors have no other conflicts of interest to declare.

Ethical Statement: The authors are accountable for all aspects of the work in ensuring that questions related to the accuracy or integrity of any part of the work are appropriately investigated and resolved. The study was 
conducted in accordance with the Declaration of Helsinki (as revised in 2013). The study was approved by institutional review board of Taipei Veterans General Hospital with the registration number of 2017-05-E01A, 2017-11E02B, 2018-03-E02C, 2019-01-E02B, 2019-03-E01B, and informed consent was taken from all the patients.

Open Access Statement: This is an Open Access article distributed in accordance with the Creative Commons Attribution-NonCommercial-NoDerivs 4.0 International License (CC BY-NC-ND 4.0), which permits the noncommercial replication and distribution of the article with the strict proviso that no changes or edits are made and the original work is properly cited (including links to both the formal publication through the relevant DOI and the license). See: https://creativecommons.org/licenses/by-nc-nd/4.0/.

\section{References}

1. 2015 Annual Pediatric Cancer Registration Report in Taiwan, Childhood Cancer Foundation of R.O.C., Republic of China in Taiwan (Updated on April 19, 2016). Available online: http://www.ccfroc.org.tw/content_sub.ph p?id=135\&level1ID=12\&level2ID=2\&level3ID=2

2. Wong TT, Ho DM, Chang KP, et al. Primary pediatric brain tumors: statistics of Taipei VGH, Taiwan (19752004). Cancer 2005;104:2156-67.

3. Sweet WH. The uses of nuclear disintegration in the diagnosis and treatment of brain tumor. N Engl J Med $1951 ; 245: 875-8$.

4. International Atomic Energy Agency (IAEA) Publication (2001). Current status of neutron capture therapy. IAEATECDOC-1223. Available online: https://pub.iaea.org/ MTCD/Publications/PDF/te_1223_prn.pdf

5. Miyatake S, Kawabata S, Yokoyama K, et al. Survival benefit of boron neutron capture therapy for recurrent malignant gliomas. J Neurooncol 2009;91:199-206.

6. Kankaanranta L, Seppälä T, Koivunoro H, et al. L-boronophenylalanine-mediated boron neutron capture therapy for malignant glioma progressing after external beam radiation therapy: a Phase I study. Int J Radiat Oncol Biol Phys 2011;80:369-76.

7. Barth RF, Zhang Z, Liu T. A realistic appraisal of boron neutron capture therapy as a cancer treatment modality. Cancer Commun (Lond) 2018;38:36.

8. Louis DN, Perry A, Reifenberger G, et al. The 2016 World Health Organization Classification of Tumors of the Central Nervous System: a summary. Acta
Neuropathol 2016;131:803-20.

9. Wang HE, Liao AH, Deng WP, et al. Evaluation of 4-borono-2-18F-fluoro-L-phenylalanine-fructose as a probe for boron neutron capture therapy in a gliomabearing rat model. J Nucl Med 2004;45:302-8.

10. Liu YH, Tsai PE, Yu HT, et al. Performance evaluation of the source description of the THOR BNCT epithermal neutron beam. Appl Radiat Isot 2011;69:1892-6.

11. Tsai YL, Tsai SC, Chen YW, et al. Efficacy of therapeutic play for pediatric brain tumor patients during external beam radiotherapy. Childs Nerv Syst 2013;29:1123-9.

12. Wen PY, Macdonald DR, Reardon DA, et al. Updated response assessment criteria for high-grade gliomas: response assessment in neuro-oncology working group. J Clin Oncol 2010;28:1963-72.

13. CTCAE ver. 5.0. Available online: https://ctep.cancer. gov/protocolDevelopment/electronic_applications/docs/ CTCAE_v5_Quick_Reference_8.5x11.pdf

14. Miyatake S, Kawabata S, Hiramatsu R, et al. Boron neutron capture therapy with bevacizumab may prolong the survival of recurrent malignant glioma patients: four cases. Radiat Oncol 2014;9:6.

15. Stupp R, Mason WP, van den Bent MJ, et al. Radiotherapy plus concomitant and adjuvant temozolomide for glioblastoma. N Engl J Med 2005;352:987-96.

16. Westphal M, Hilt DC, Bortey E, et al. A phase 3 trial of local chemotherapy with biodegradable carmustine (BCNU) wafers (Gliadel wafers) in patients with primary malignant glioma. Neuro Oncol 2003;5:79-88.

17. Mun EJ, Babiker HM, Weinberg U, et al. Tumor-treating fields: a fourth modality in cancer treatment. Clin Cancer Res 2018;24:266-75.

18. Sauerwein W, Wittig A, Moss R, et al. Neutron Capture Therapy. New York: Springer, 2012:41-54.

19. Locher GL. Biological effects and therapeutical possibilities of neutrons. Am J Roentgenol Radium Ther 1936;36:1-13.

20. Barth RF, Vicente MG, Harling OK, et al. Current status of boron neutron capture therapy of high grade gliomas and recurrent head and neck cancer. Radiat Oncol 2012;7:146.

21. Kawabata S, Miyatake S, Nonoguchi N, et al. Survival benefit from boron neutron capture therapy for the newly diagnosed glioblastoma patients. Appl Radiat Isot 2009;67:S15-8.

22. Nakagawa Y, Kageji T, Mizobuchi Y, et al. Clinical results of BNCT for malignant brain tumors in children. Appl Radiat Isot 2009;67:S27-30. 
23. Coderre JA, Makar MS, Micca PL, et al. Derivations of relative biological effectiveness for the high-let radiations produced during boron neutron capture irradiations of the 9L rat gliosarcoma in vitro and in vivo. Int $\mathrm{J}$ Radiat Oncol Biol Phys 1993;27:1121-9.

24. Chinot OL, Wick W, Mason W, et al. Bevacizumab plus radiotherapy-temozolomide for newly diagnosed glioblastoma. N Engl J Med 2014;370:709-22.

25. Furuse M, Kawabata S, Kuroiwa T, et al. Repeated treatments with bevacizumab for recurrent radiation necrosis in patients with malignant brain tumors: a report of 2 cases. J Neurooncol 2011;102:471-5.

doi: $10.21037 /$ tro-20-20

Cite this article as: Chen YW, Lan TL, Lee YY, Lin KH, Liang ML, Chen HH, Pan PS, Chang FC, Wu CC, Lin SC, Kang YM, Lee JC, Liu HM, Chen KH, Chen JK, Lin CF, Kuo YC, Hsu SM, Huang WS, Chou FI. Using precise boron neutron capture therapy as a salvage treatment for pediatric patients with recurrent brain tumors. Ther Radiol Oncol 2020;4:30.
26. Lin KH, Chen YW, Lee RC, et al. Nuclear theranostics in Taiwan. Nucl Med Mol Imaging 2019;53:86-91.

27. Watabe T, Ikeda H, Nagamori S, et al. 18F-FBPA as a tumor-specific probe of L-type amino acid transporter 1 (LAT1): a comparison study with $18 \mathrm{~F}-\mathrm{FDG}$ and 11C-Methionine PET. Eur J Nucl Med Mol Imaging 2017;44:321-31.

28. Maxwell R, Luksik AS, Garzon-Muvdi T, et al. Populationbased Study Determining Predictors of Cancer-Specific Mortality and Survival in Pediatric High-grade Brainstem Glioma. World Neurosurg 2018;119:e1006-15. 